\title{
Die Analyse von Nebenwirkungen von Coaching für Klienten aus einer qualitativen Perspektive
}

\author{
Carsten C. Schermuly ${ }^{1}$ Carolin Graßmann ${ }^{1}$
}

Online publiziert: 17. August 2016

(c) Der/die Autor(en) 2016. Dieser Artikel ist eine Open-Access-Publikation.

Zusammenfassung Die Forschung zu Nebenwirkungen von Coaching für Klienten schreitet voran. Es liegen mittlerweile Ergebnisse darüber vor, wie häufig Nebenwirkungen von Coaches und Klienten wahrgenommen werden und welche Ursachen für diese verantwortlich sind. In diesem Artikel wird zunächst der aktuelle Stand der Forschung zu Nebenwirkungen von Coaching für Klienten dargestellt. Diese Forschung ist sehr stark quantitativ geprägt. Deswegen wird nach einer Literaturübersicht ein qualitativer Ansatz gewählt und acht Coachingfälle werden detailliert und systematisch vorgestellt, in denen eine Nebenwirkung aufgetreten ist. Durch die breite Darstellung wird es möglich zu erfassen, wie Nebenwirkungen in Coachings konkret auftreten. Danach werden die kognitiven und affektiven Reaktionen der Coaches und Klienten bezogen auf die Nebenwirkungen sowie die Bewältigungsstrategien der Coaches analysiert. Die Ergebnisse zeigen, dass insbesondere Nebenwirkungen auftreten, die soziale Konsequenzen haben. Mehrheitlich werden die Nebenwirkungen als nicht notwendig für die Zielerreichung angesehen, doch können diese innerhalb des Coachings bewältigt werden.

Bewältigung $\cdot$ Coaching $\cdot$ Nebenwirkungen $\cdot$ Negative Effekte · Verhalten

Carsten C. Schermuly

carsten.schermuly@ srh-hochschule-berlin.de

1 SRH Hochschule Berlin, Ernst-Reuter-Platz 10, 10587 Berlin, Deutschland

\section{The Analysis of Side Effects of Coaching for Clients From a Qualitative Perspective}

\begin{abstract}
Research on the side effects of coaching for clients is growing. By now, we know how often coaches and clients experience side effects and which causes are responsible for their emergence. In this article, we present a literature review about side effects of coaching for clients, which is dominated by quantitative study designs. Moreover, we use a qualitative research design to systematically examine eight coaching cases in which side effects occurred. These cases display in more depth how side effects arise in coaching. Subsequently, we analyze coaches' and clients' cognitive and affective reactions towards side effects and coaches' coping strategies. Findings show that most side effects have social consequences. The majority of side effects were not necessary for goal attainment, but could be managed during coaching.
\end{abstract}

Coping $\cdot$ Coaching $\cdot$ Side effects $\cdot$ Negative effects · Behavior

\section{Einleitung}

Business-Coaching ist ein dyadischer Beratungsprozess zwischen einem Klienten und einem professionellen Coach, um arbeitsbezogene Anliegen zu bearbeiten (Feldman und Lankau 2005; Theeboom et al. 2014). Coaching soll dabei nicht nur die Arbeitsleistung und Zufriedenheit des Klienten verbessern, sondern auch dadurch die Effektivität der Organisation des Klienten erhöhen (Kilburg 1996). Auch wenn der Stand der Forschung noch als unzureichend angesehen werden kann (Möller und Kotte 2011), werden positive Effekte von Coaching zunehmend von der 
empirischen Forschung bestätigt. So konnten bereits mehrere Metaanalysen überblicksartig zeigen, dass Coaching zu einem breiten Spektrum an wünschenswerten Effekten führen kann. Mittelstarke positive Effekte wurden auf die selbstregulatorischen Fähigkeiten des Klienten, dessen Leistung, Einstellung und Wohlbefinden sowie auf die Coping-Fähigkeiten des Klienten nachgewiesen (Theeboom et al. 2014). In einer weiteren Metaanalyse konnte gezeigt werden, dass mit Hilfe von Coaching sogar größere und konsistentere positive Effekte erzielt werden können als mit anderen populären Interventionen wie beispielsweise dem Multi-Source-Feedback (Jones et al. 2015; für einen Vergleich der Metaanalysen im Coaching siehe Kotte et al. 2016).

Allerdings sind nicht nur erwünschte Effekte von Coaching denkbar und bekannt (siehe zum Beispiel erste Arbeiten von Kilburg 2002 oder Seiger und Künzli 2011). Unerwünschte Erfahrungen sind ein natürlicher Bestandteil von engen Beziehungen (Duck und Wood 1995), wozu sich auch die Coachingbeziehung zwischen Coach und Klient zählen lässt. Im Gegensatz zu den erwünschten Effekten haben Nebenwirkungen von Coaching bisher wenig Beachtung in der Coaching-Forschung und -Praxis erhalten. Dies steht im Gegensatz zu benachbarten Disziplinen wie dem Mentoring, der Supervision oder auch der Psychotherapie, die sich bereits seit längerer Zeit mit dieser Thematik beschäftigen (z. B. Eby und Allen 2002; Mohr 1995; RamosSánchez et al. 2002).

Es erscheint in mehrfacher Hinsicht für die Coachingforschung und Coachingpraxis sinnvoll und notwendig, das vollständige Wirkungsspektrum von Coaching zu beleuchten und damit auch Nebenwirkungen Aufmerksamkeit zu schenken. Nur wenn Wissen über Nebenwirkungen existiert, können Klienten darüber aufgeklärt werden, welches Spektrum an Effekten durch ein Coaching zu erwarten ist. Zum Zweiten kann Wissen über Nebenwirkungen dazu beitragen, diese erfolgreich zu bewältigen oder gar positiv zu nutzen. Zum Dritten kann die Beschäftigung mit Nebenwirkungen zur weiteren Professionalisierung von Coaching beitragen, welche durch evidenzbasierte Forschung vorangetrieben werden kann (Bennett 2006).

Im Folgenden soll zunächst der aktuelle Stand der Forschung zu Nebenwirkungen von Coaching für Klienten dargestellt werden. Diese Forschung ist sehr stark quantitativ geprägt. Deswegen wird nach einer Literaturübersicht ein qualitativer Ansatz gewählt und verschiedene Coachingfälle detailliert und systematisch vorgestellt, in denen Nebenwirkungen aufgetreten sind. Die Studie soll dazu beitragen, im Einzelfall zu verstehen, (1) wie sich Nebenwirkungen in der Coaching-Praxis konkret zeigen können, (2) wie Klienten und Coaches darauf reagieren und (3) wie Coaches es in der Praxis geschafft haben, aufgetretene Nebenwirkungen zu bewältigen.

\section{Forschungsstand zu Nebenwirkungen von Coaching}

\subsection{Was sind Nebenwirkungen von Coaching?}

Zunächst soll definiert werden, was unter Nebenwirkungen von Coaching zu verstehen ist. Nebenwirkungen von Coaching werden definiert als ,,alle für den Klienten schädlichen oder unerwünschten Folgen, die unmittelbar durch das Coaching verursacht werden und parallel dazu oder im Anschluss daran auftreten. Diese Effekte sind unbeabsichtigt, schädlich und direkt verbunden mit dem Coachingprozess" (Schermuly et al. 2014, S. 19). Diese Definition bezieht sich auf den Klienten. Nebenwirkungen können auch für den Coach (Schermuly 2014) und für die Organisation (Oellerich 2016) entstehen ${ }^{1}$; diese werden in dieser Definition aber nicht berücksichtigt. Klar zu trennen sind Nebenwirkungen auch vom Misserfolg eines Coachings. Nebenwirkungen können sowohl in nicht erfolgreichen Coachings auftreten als auch in erfolgreichen. Misserfolg bezieht sich darauf, dass die gemeinsam vereinbarten Ziele des Coachings nicht erreicht werden konnten (Kilburg 2002). Nebenwirkungen sind nicht zwingend an die Zielerreichung gekoppelt. Sie können auch auftreten, wenn alle Ziele des Coachings erreicht wurden. Langfristig ist es sogar in dieser Konzeption vorstellbar, dass eine Nebenwirkung positive Konsequenzen haben kann.

Ein Beispiel zur Verdeutlichung: Ein Klient, ein Spezialist in der internen Unternehmensberatung eines großen Handelsunternehmens, möchte in einem Coaching seinen nächsten Karriereschritt planen. Der Coach unterstützt ihn bei diesem Ziel, aber der Klient schafft es zunächst nicht, sich neue mögliche Karriereperspektiven zu eröffnen (vorläufiger Misserfolg). In weiteren Gesprächen werden die Ressourcen des Klienten aktiviert und er erkennt zunehmend besser, welcher Karriereschritt seinen Vorstellungen am besten entspricht (Erfolg). Nun wird der Klient allerdings zunehmend unzufriedener mit seiner aktuellen Posi-

\footnotetext{
1 Nebenwirkungen für Coaches treten deutlich häufiger auf als solche für Klienten. Die häufigsten Effekte für Coaches sind die Enttäuschung, dass die Langzeitwirkung des Coachings nicht beobachtet werden konnte, die persönliche Betroffenheit durch ein Thema oder die Angst der Coachrolle nicht gerecht zu werden. Sie treten in mehr als $40 \%$ der untersuchten Coachingfälle auf. Die Nebenwirkungen sind mit erhöhtem Stresserleben und emotionaler Erschöpfung sowie geringerem Kompetenzgefühl und mehr Ohnmachtserleben seitens der Coaches assoziiert (Schermuly, 2014).

Zentrale negative Effekte für die Organisation sind beispielsweise eine als zu stark empfundene Entwicklung des Klienten, die zu Schwierigkeiten im beruflichen Umfeld führt, Kündigungen und eine nachhaltige Verunsicherung des Klienten. Als wichtigste Ursache wird die Einstellung der Organisation zu Coaching benannt. Aber auch die unzureichende Auftragsklärung, fehlende Motivation des Klienten sowie die Arbeitsweise des Coaches werden als Ursachen für negative Effekte für die Organisation gesehen (Oellerich 2016).
} 
tion, da diese ihm nicht mehr genügt (Nebenwirkung). Er empfindet das Verharren in der derzeitigen Position als unangenehm. Dieses Beispiel verdeutlicht die drei Bestandteile der Definition von Nebenwirkungen: Die Nebenwirkung wurde direkt durch das Coaching hervorgerufen, ist unbeabsichtigt aufgetreten und wird durch den Klienten zumindest kurzfristig als negativ angesehen. Langfristig könnte aber die Unzufriedenheit dazu führen, dass der Klient mehr Energie in einen Stellenwechsel investiert und dadurch in einer anderen Position glücklicher wird.

\subsection{Nebenwirkungen für Klienten aus der Sicht von Coaches}

Bisher existieren nur wenige Studien, die sich mit Nebenwirkungen von Coaching beschäftigen. In einer Marktbefragung (Seiger und Künzli 2011) geben 17,2\% der Coaches an, dass sie schon mit unerwünschten Effekten auf Seiten ihrer Klienten konfrontiert waren. Schermuly et al. (2014) haben in ihrer ersten explorativen Studie, die eben vorgestellte Definition 21 erfahrenen Coaches vorgestellt und offen in einem Telefoninterview erfragt, welche Neben- wirkungen die Coaches bisher erlebt haben und welche sie sich darüber hinaus vorstellen könnten. Die Antworten der Coaches wurden daraufhin in Kategorien zusammengefasst. Hieraus entstand eine Liste mit potenziellen Nebenwirkungen für den Klienten. Ebenso erfragt wurden Ursachen, die zur Entstehung dieser Nebenwirkungen beigetragen haben.

Im nächsten Schritt wurden 123 Coaches quantitativ befragt, wie oft diese Nebenwirkungen in ihrem zuletzt abgeschlossenen Coaching für den Klienten aufgetreten sind und welche Ursachen sie für diese sahen. In über der Hälfte der Coachings (57\%) berichteten die Coaches mindestens eine Nebenwirkung. Durchschnittlich wurden 2,1 Nebenwirkungen pro Coaching berichtet. Die Intensität der Nebenwirkung schätzten die Coaches eher als gering und die Dauer als eher kurz (kürzer als vier Wochen) ein. Die untersuchten Coachings wurden nichtdestotrotz als sehr erfolgreich bewertet. Nebenwirkungen traten demnach auch in erfolgreichen Coachings häufig auf.

Die drei häufigsten Nebenwirkungen, die von den Coaches berichtet wurden, sind das Anstoßen von Problemen, die nicht bearbeitet werden konnten (26,0\%), die Abwandlung ursprünglicher Ziele, ohne dass der Klient/die Klientin

Tab. 1 Kategorien negativer Effekte von Coaching für Klienten

\begin{tabular}{|c|c|c|c|c|c|}
\hline $\begin{array}{l}\text { Psychisches Wohl- } \\
\text { befinden }(\mathrm{C}, \mathrm{K} \text { in } \\
\%)\end{array}$ & $\begin{array}{l}\text { Soziale Integration } \\
(\mathrm{C}, \mathrm{K} \text { in } \%)\end{array}$ & $\begin{array}{l}\text { Leistungsfähigkeit } \\
(\mathrm{C}, \mathrm{K} \text { in \%) }\end{array}$ & $\begin{array}{l}\text { Bewertung der Ar- } \\
\text { beitsrolle }(\mathrm{C}, \mathrm{K} \text { in } \\
\%)\end{array}$ & $\begin{array}{l}\text { Materielle Verluste } \\
(\mathrm{C}, \mathrm{K} \text { in } \%)\end{array}$ & $\begin{array}{l}\text { Sonstige }(\mathrm{C}, \mathrm{K} \text { in } \\
\%)\end{array}$ \\
\hline $\begin{array}{l}\text { Anstoßen von Pro- } \\
\text { blemen, die nicht } \\
\text { bearbeitet werden } \\
\text { konnten (C: } 26 ; \mathrm{K} \text { : } \\
\text { 23) }\end{array}$ & $\begin{array}{l}\text { Verschlechterung } \\
\text { Beziehung: Vorge- } \\
\text { setzter } \\
\text { (C: 14; K: 18) }\end{array}$ & $\begin{array}{l}\text { Schwankungen der } \\
\text { Arbeitsleistung } \\
(\mathrm{C}: 13 ; \mathrm{K}: 20)\end{array}$ & $\begin{array}{l}\text { Bedeutsamkeitsverlust } \\
\text { ggü. Arbeit } \\
\text { (C: 17; K: 29) }\end{array}$ & $\begin{array}{l}\text { Arbeitgeberwechsel } \\
\text { mit schlechteren } \\
\text { Arbeitsbedingungen } \\
\text { (C: } 3 ; \mathrm{K}: 5)\end{array}$ & $\begin{array}{l}\text { Abwandlung von } \\
\text { Zielen gegen den } \\
\text { Willen des Klienten } \\
\text { (C: 17; K: 23) }\end{array}$ \\
\hline $\begin{array}{l}\text { Reduktion Lebens- } \\
\text { zufriedenheit } \\
\text { (C: } 10 ; \mathrm{K}: 22)\end{array}$ & $\begin{array}{l}\text { Abhängigkeits- } \\
\text { verhältnis ggü. } \\
\text { Coach } \\
\text { (C: } 12 ; \mathrm{K}: 10)\end{array}$ & $\begin{array}{l}\text { Reduktion Arbeits- } \\
\text { motivation } \\
(\mathrm{C}: 9 ; \mathrm{K}: 22)\end{array}$ & $\begin{array}{l}\text { Reduktion Arbeits- } \\
\text { zufriedenheit } \\
\text { (C: 13; K: 32) }\end{array}$ & $\begin{array}{l}\text { Arbeitsplatzverlust } \\
\text { (C: } 2 ; \mathrm{K}: 4)\end{array}$ & $\begin{array}{l}\text { Rechtsstreit mit } \\
\text { Coach } \\
(\mathrm{C}: 1 ; \mathrm{K}: 0)\end{array}$ \\
\hline $\begin{array}{l}\text { Verschlechterung } \\
\text { Work-Life-Balance } \\
\text { (C: } 9 ; \mathrm{K}: 20)\end{array}$ & $\begin{array}{l}\text { Verschlechterung } \\
\text { Beziehung: Ehepart- } \\
\text { ner } \\
(\mathrm{C}: 6 ; \mathrm{K}: 6)\end{array}$ & $\begin{array}{l}\text { Verschlechterung } \\
\text { der Arbeitsleistung } \\
(\mathrm{C}: 4 ; \mathrm{K}: 15)\end{array}$ & $\begin{array}{l}\text { Reduktion Kompe- } \\
\text { tenzerleben } \\
\text { (C: } 10 ; \mathrm{K}: 17)\end{array}$ & $\begin{array}{l}\text { Finanziell bedroh- } \\
\text { liche Situation } \\
(\mathrm{C}: 2 ; \mathrm{K}: 5)\end{array}$ & $\begin{array}{l}\text { Persönliche Infor- } \\
\text { mationen an Dritte } \\
(\mathrm{C}: 1 ; \mathrm{K}: 6)\end{array}$ \\
\hline $\begin{array}{l}\text { Arbeitsplatzangst } \\
\text { (C: } 7 ; \mathrm{K}: 5)\end{array}$ & $\begin{array}{l}\text { Verschlechterung } \\
\text { Beziehung: andere } \\
\text { Familienmitglieder } \\
\text { (C: } 6 ; \mathrm{K}: 10)\end{array}$ & - & $\begin{array}{l}\text { Reduktion Einfluss- } \\
\text { erleben (C: 4; K: 7) }\end{array}$ & - & - \\
\hline $\begin{array}{l}\text { Verschlimmerung } \\
\text { psychische Störung } \\
(\mathrm{C}: 2 ; \mathrm{K}: 5)\end{array}$ & $\begin{array}{l}\text { Verschlechterung } \\
\text { Beziehung: Kolle- } \\
\text { gen } \\
\text { (C: } 5 ; \mathrm{K}: 16)\end{array}$ & - & $\begin{array}{l}\text { Reduktion Selbstbe- } \\
\text { stimmungserleben } \\
(\mathrm{C}: 4 ; \mathrm{K}: 6)\end{array}$ & - & - \\
\hline $\begin{array}{l}\text { Entwicklung psychi- } \\
\text { sche Störung } \\
(\mathrm{C}: 2 ; \mathrm{K}: 2)\end{array}$ & $\begin{array}{l}\text { Verschlechterung } \\
\text { Beziehung: Mitar- } \\
\text { beiter } \\
\text { (C: } 2 ; \mathrm{K}: 13)\end{array}$ & - & - & - & - \\
\hline $\begin{array}{l}\text { Mehr Konsum von } \\
\text { Zigaretten, Alkohol } \\
\text { oder Medikamenten } \\
(\mathrm{C}: 2 ; \mathrm{K}: 5)\end{array}$ & - & - & - & - & - \\
\hline
\end{tabular}

$C$ Häufigkeit der Nebenwirkung aus der Perspektive der Coaches (Schermuly et al. 2014), $K$ Häufigkeit der Nebenwirkung aus der Perspektive der Klienten (Graßmann und Schermuly, 2016) 
das wollte $(17,1 \%)$ und dass der Klient/die Klientin seine/ihre Arbeit als weniger bedeutsam erlebt hat $(17,1 \%)$. Schwerwiegende Nebenwirkungen, wie beispielsweise die Entstehung von Symptomen einer psychischen Störung $(1,6 \%)$ oder deren Verschlechterung $(2,4 \%)$ treten eher selten auf. Ein Überblick über die berichteten Häufigkeiten kann Tab. 1 entnommen werden. Schermuly (2016) nahm eine Kategorisierung der Nebenwirkungen vor. Die größte Kategorie bilden Nebenwirkungen im Bereich des psychischen Wohlbefindens. $\mathrm{Zu}$ dieser Kategorie zählt u. a. die Reduktion der Lebenszufriedenheit, die Verschlechterung der Work-Life-Balance oder auch das Auftreten oder die Verschlimmerung von Symptomen einer psychischen Störung. Des Weiteren können sich auch Nebenwirkungen im Bereich der sozialen Integration zeigen, beispielsweise als eine verschlechterte Beziehung zu Vorgesetzten, Kollegen, Mitarbeitern, zum Ehepartner oder anderen Familienmitgliedern. Auch kann sich ein Abhängigkeitsverhältnis zum Coach herausbilden. In die Kategorie Leistungsfähigkeit fällt das Absinken oder ein stärkeres Schwanken der eigenen Arbeitsleistung, aber auch der Verlust an Arbeitsmotivation. Daneben kann sich Coaching auch auf die Bewertung der Arbeitsrolle auswirken, z. B. in Form eines Bedeutsamkeitsverlustes gegenüber der eigenen Arbeit oder der reduzierten Arbeitszufriedenheit. Zur Kategorie der materiellen Verluste gehört beispielsweise der eher seltene, ungewollte Arbeitsplatzverlust oder auch eine finanziell bedrohliche Situation. Sonstige Nebenwirkungen können darüber hinaus einen Rechtstreit mit dem Coach beinhalten oder auch die Weitergabe von persönlichen Informationen an Dritte.

Als verantwortlich für die Entstehung von Nebenwirkungen sahen Coaches vor allem die Klienten an. Die Coaches berichteten, dass sie die Ursache in einem geringen Problembewusstsein sahen (23\%), in falschen Erwartungen $(20 \%)$, einer psychischen Vorerkrankung $(18 \%)$, einem unkonkreten Coachingziel $(16 \%)$ oder dem Vorenthalten wichtiger Informationen (12\%). Auch die Organisation kann aus Sicht der Coaches zum Entstehen von Nebenwirkungen beitragen: durch ein schlechtes Transferumfeld $(16 \%)$, zu wenig Unterstützung durch die Organisation (10\%) oder dadurch, dass die Organisation das Coaching erzwungen hat (10\%). Zusätzlich wurde von den Autoren eine Kategorie Sonstiges genutzt. In $24 \%$ aller Coachings mit Nebenwirkung wurde zu wenig Zeit als Ursache benannt. Weitere wahrgenommene Ursachen waren zu wenig finanzielle Mittel (18\%) sowie eine unzureichende Diagnostik (17\%). In geringerem Maße sahen die Coaches die Ursache in ihrer eigenen Arbeit begründet: keine Supervision $(11 \%)$, zu wenig Wissen über die Arbeit/Organisation des Klienten (11\%), zu wenig fachliche Expertise (11\%) sowie die eigene Überarbeitung (9\%). Darüber hinaus zeigte sich ein Zusammenhang zwischen der Anzahl der be- arbeiteten Themen und der Anzahl der Nebenwirkungen: Umso mehr Themen angerissen wurden, umso mehr Nebenwirkungen wurden berichtet.

\subsection{Nebenwirkungen aus der Sicht von Klienten}

Die explorative Studie von Schermuly et al. (2014) bietet neben einer Definition auch eine Übersicht an möglichen Nebenwirkungen für den Klienten. Da in dieser Studie zunächst Coaches befragt wurden, blieb offen, wie häufig die Klienten selbst Nebenwirkungen wahrnahmen und welche Ursache sie für das Auftreten sahen. Graßmann und Schermuly (2016) befragten hierfür die Klienten zu zwei Messzeitpunkten. Es wurden 111 Klienten befragt, die innerhalb der vergangenen zwölf Monate ein Coaching abgeschlossen hatten. Nach weiteren acht Wochen nahmen noch einmal 42 Klienten an der Follow-up Befragung teil. Die Auftretenshäufigkeiten der Nebenwirkungen lagen etwas höher als in der Studie von Schermuly et al. (2014). In $68 \%$ der Coachings trat mindesten eine Nebenwirkung auf. Durchschnittlich waren es 3,1 Nebenwirkungen pro Coaching. Die Rangfolge der Nebenwirkungen ist zwischen den beiden Studien ähnlich (Spearmans Rho $=0,87 ; p<0,01$ ). Häufig von den Coaches wahrgenommene Nebenwirkungen landeten auch in der Klientenstichprobe auf den vorderen Rängen. Die drei häufigsten Nebenwirkungen aus der Sicht der Klienten waren die reduzierte Arbeitszufriedenheit (32\%), der Bedeutsamkeitsverlust gegenüber der eigenen Arbeit (29\%) sowie die Abwandlung der Ziele ohne dass der Klient/die Klientin das wollte (23\%). Die Übersicht der Häufigkeiten aus Sicht der Klienten kann ebenfalls Tab. 1 entnommen werden.

Des Weiteren wurde untersucht, welche Coachingvariablen einen Einfluss auf die Anzahl der Nebenwirkungen hatten. Hierfür wurden die Klienten zum ersten Messzeitpunkt darum gebeten, die Beziehungsqualität zu ihrem Coach, dessen wahrgenommene Expertise und ihre eigene Veränderungsmotivation einzuschätzen. Die Ergebnisse zum ersten Messzeitpunkt zeigen, dass die Beziehungsqualität zwischen Coach und Klient ein starker Prädiktor für die Anzahl der Nebenwirkungen ist. Je besser die Beziehungsqualität von den Klienten eingeschätzt wurde, desto weniger Nebenwirkungen wurden berichtet. Dies gilt ebenso für die wahrgenommene Expertise des Coaches: Umso höher der Klient die Expertise des Coaches eingeschätzt hat, umso weniger Nebenwirkungen wurden wahrgenommen. Es konnte allerdings kein Einfluss der Veränderungsmotivation des Klienten auf die Nebenwirkungen nachgewiesen werden.

Zum zweiten Messzeitpunkt wurden noch einmal die Nebenwirkungen erhoben. Es zeigte sich derselbe Einfluss der Beziehungsqualität auf die Anzahl der Nebenwirkungen, was die Annahme stützt, dass die Beziehungsqualität zwi- 
schen Coach und Klient ein sehr bedeutsamer Prädiktor für die Wirkung von Coaching ist (Baron und Morin 2009; Bluckert 2005; de Haan et al. 2013). Die durch den Klienten wahrgenommene Expertise des Coaches besitzt allerdings keine Vorhersagekraft für die Nebenwirkungen zum zweiten Messzeitpunkt.

\subsection{Forschungslücke: Qualitative Analyse von Nebenwirkungsfällen}

Diese vorgestellten Ergebnisse bieten einen ersten Einblick, welche Nebenwirkungen durch Coaching auftreten können, wie häufig und intensiv Coaches und Klienten diese erleben und welche Ursachen für das Auftreten verantwortlich sein können. Es bleibt allerdings unbeantwortet, wie sich diese Nebenwirkungen in einem Coachingprozess konkret manifestieren und welche Auswirkungen Sie auf den Coachingprozess haben. Bennett (2006) schlägt für die weitere Coachingforschung vor, dass sowohl quantitative als auch qualitative Forschung benötigt wird. So bleibt durch die bisherige eher quantitativ orientierte Forschung zu Nebenwirkungen beispielsweise unklar, wie Coach und Klient konkret auf das Auftreten einer Nebenwirkung reagieren. Um mögliche Ansatzpunkte zu finden, mit denen Nebenwirkungen bewältigt werden können, ist es notwendig ein besseres Verständnis über das Erleben im Einzelfall zu ent- wickeln. Hierzu zählen sowohl die kognitive als auch die affektive Reaktion der Beteiligten. Was denkt und fühlt der Klient, wenn er oder sie eine Nebenwirkung wahrnimmt? Was denkt und fühlt der Coach als Partner der Coachingbeziehung? Neben den Gedanken und Gefühlen der Beteiligten ist auch der behaviorale Aspekt noch nicht geklärt: Wie verhalten sich Coach und Klient nach dem Auftreten einer Nebenwirkung? Klienten berichten häufiger über Nebenwirkungen als Coaches (Graßmann und Schermuly, 2016). Es bleibt demnach fraglich, ob Klienten diese Effekte im Coaching offen thematisieren. Weiterhin sollte geklärt werden, wie diese Nebenwirkungen reduziert werden können und welche Strategien sich hierfür in der Praxis als hilfreich herausstellen. Somit kristallisieren sich drei zentrale Fragen heraus:

Frage 1: Wie zeigen sich Nebenwirkungen von Coaching konkret in der Praxis?

Frage 2: Wie reagieren Coach und Klient, wenn eine Nebenwirkung auftritt?

Frage 3: Wie können Nebenwirkungen bewältigt werden?

Tab. 2 Interviewphasen und Hauptfragen

\begin{tabular}{|c|c|c|}
\hline & Phase & Hauptfragen \\
\hline 1 & Begrüßung & - \\
\hline 2 & Demografische Variablen Coach & z. B. Alter, Berufserfahrung, Coachings pro Jahr \\
\hline 3 & $\begin{array}{l}\text { Vorstellung der Definition von Nebenwir- } \\
\text { kungen und Abfrage des Auftretens }\end{array}$ & $\begin{array}{l}\text { Haben Sie in einem Ihrer vergangenen Coachings erlebt, dass ein negativer Effekt } \\
\text { gemäß dieser Definition aufgetreten ist? }\end{array}$ \\
\hline 4 & $\begin{array}{l}\text { Präzision der Nebenwirkung und des } \\
\text { Coachings }\end{array}$ & $\begin{array}{l}\text { z. B. Um welchen negativen Effekt handelte es sich genau? } \\
\text { Wie äußerte sich der Effekt? } \\
\text { Konnte das Coaching, Ihrer Meinung nach, erfolgreich abgeschlossen werden? } \\
\text { In welcher zeitlichen Phase des Coachings ist der negative Effekt aufgetreten? } \\
\text { Wie lange war der negative Effekt präsent? } \\
\text { Was war das Ziel/die Ziele des betreffenden Coachings? }\end{array}$ \\
\hline 5 & Reaktionen & $\begin{array}{l}\text { z. B. Welche Gefühle und Gedanken hatten Sie, als Sie den negativen Effekt erkannt } \\
\text { haben? } \\
\text { Welche Gefühle und Gedanken hat der Coachee gezeigt, als der Coachee Ihnen von } \\
\text { dem negativen Effekt berichtet hat? }\end{array}$ \\
\hline 6 & Ursachen & Keine Berücksichtigung \\
\hline 7 & Umgang mit Nebenwirkung & $\begin{array}{l}\text { z. B. Inwiefern konnte der negative Effekt genutzt werden, um andere positive Wirkun- } \\
\text { gen zu erzielen? } \\
\text { War das Auftreten des negativen Effektes von Nöten, um das Coaching erfolgreich } \\
\text { abzuschließen? } \\
\text { Konnten Sie den aufgetretenen negativen Effekt reduzieren? } \\
\text { Welche Verhaltensweisen haben Sie gezeigt, die zur Reduktion des negativen Effektes } \\
\text { beigetragen hat? } \\
\text { Welches Verhalten hat der Coachee gezeigt, das zur Reduktion des negativen Effekts } \\
\text { beigetragen hat? } \\
\text { Welche Rahmenbedingungen haben die Reduktion des negativen Effektes unterstützt? }\end{array}$ \\
\hline 8 & Demografische Variablen Klient & Alter und Geschlecht \\
\hline 9 & Verabschiedung und Dank & - \\
\hline
\end{tabular}




\section{Methode}

Zur Beantwortung dieser Fragen wurde ein qualitativer Ansatz gewählt. Der qualitative Ansatz birgt das Potenzial den individuellen Coachingfall besser zu verstehen, indem das Individuum mit seinen Erleben als Ganzes stärker in den Fokus rückt (Brüsemeister 2008). Dadurch können detailliertere Informationen gewonnen werden, weil man sich auf das subjektive Erleben und nicht auf die Durchschnittswerte bezieht (Brüsemeister 2008). Zudem ist dieser Ansatz geeignet für das explorative Vorgehen, welches aufgrund der wenigen Kenntnisse zu diesen Fragestellungen indiziert ist.

\subsection{Vorgehen}

Im Frühjahr 2014 wurden von einer Masterandin ${ }^{2} 50$ Coaches aus ganz Deutschland zufällig ausgewählt und per E-Mail angeschrieben. 19 Coaches nahmen an der Studie teil (Rücklaufrate $=38 \%$ ). Mit jedem Coach wurde ein Telefoninterview über jeweils einen Fall geführt. Das bedeutet, dass jeder Coach nur mit einem Interview in den Ergebnissen abgebildet ist. Die Interviews zum Thema Nebenwirkungen wurden mit der Critical Incident Technique geführt (CIT; Flanagan 1954). Die CIT ist eine halbstandardisierte Interviewtechnik. Die Prinzipien lassen sich flexibel auf den jeweiligen Untersuchungsgegenstand anpassen. Kritisch ist ein Ereignis ,if it makes a ,significant " contribution, either positively or negatively, to the general aim of the activity" (Flanagan 1954, S. 338). Das Auftreten einer Nebenwirkung sehen wir als ein kritisches Ereignis für einen Coachingprozess.

Zunächst wurde der Interviewleitfaden vorgetestet. Dafür wurden neun Interviews durchgeführt. Auf Basis der Erkenntnisse aus der Vortestung wurde der Leitfaden angepasst. Die finale Version bestand aus acht Abschnitten, die in Tab. 2 vorgestellt werden. Nach der Begrüßung wurden die demografischen Variablen des Coaches erfragt. In der nächsten Phase wurden die Nebenwirkung und das Coaching, in dem sie aufgetreten ist, präzisiert. In der vierten Phase wurde mit den Fragen die Reaktionen des Coaches und des Klienten auf die Nebenwirkung ermittelt. Daran schlossen sich Fragen zu den Ursachen der Nebenwirkung an, die keinen Schwerpunkt des Artikels bilden und deswegen unberücksichtigt bleiben. In der sechsten Phase wurde die Bewältigung der Nebenwirkung thematisiert. Das Interview endete mit der Abfrage demografischer Variablen des Klienten und der Verabschiedung.

\footnotetext{
2 Wir danken Theresa Pogge für die Zusammenarbeit im Rahmen des Projektes und der Durchführung und Transkription der Interviews. Eine Darstellung der Interviews erfolgt bei Pogge (2015).
}

\subsection{Auswahl der Interviews und Auswertungsstrategie}

Es wurden insgesamt 19 Interviews durchgeführt, die alle transkribiert wurden. Um prägnante Fälle möglichst detailliert darstellen zu können, haben wir uns für eine reduzierte Auswahl entschieden. Deswegen musste eine möglichst objektive Auswahl getroffen werden. Dem Leser sollen mit dem Artikel Fallbeispiele vorgestellt werden, die möglichst repräsentativ für den Forschungsgegenstand sind. Deswegen bewerteten die Autoren jede Nebenwirkung inwieweit sie repräsentativ für die Definition nach Schermuly et al. (2014) ist. Dies wurde unabhängig voneinander auf einer Skala von 1 (=überhaupt nicht repräsentativ) bis 9 (=voll und ganz repräsentativ) vollzogen. Die unjustierte Intraklassenkorrelation offenbarte mit einem Wert von .90 eine hohe Beobachterübereinstimmung zwischen den beiden Bewertern. In einem nächsten Schritt wurde der Mittelwert für jedes Interview gebildet. Es resultierten vier Interviews mit perfekter $(M=9,0)$ und vier mit fast perfekter Repräsentativität $(M=8,5)$. Diese acht Interviews wurden ausgewählt und bilden die Grundlage der Auswertungen.

Die ausgewählten Interviews wurden im Zuge der Auswertungen mehrmals von beiden Autoren gelesen. Für die Fragestellung relevante Abschnitte wurden markiert und diskutiert. Um einen Überblick über Muster und Unterschiedlichkeiten zu erhalten, wurden die Fälle systematisch hinsichtlich der folgenden Aspekte ausgewertet: Nebenwirkungsart, Coachinganlass, Coachingerfolg, Thematisierung, Notwendigkeit und positive Nutzung der Nebenwirkung, Auftretensphase, Dauer und Reduktion der Nebenwirkung (siehe Tab. 3). Um ein besseres Verständnis für die jeweiligen Nebenwirkungen zu erreichen, sollen diese im Ergebnisteil zusätzlich breit beschrieben werden. Dabei soll mit Zitaten und sinngemäßen Wiedergaben (Paraphrasen) auf die Interviewquellen direkt Bezug genommen werden. Zitiert werden die Transkripte, die im Anhang der Masterarbeit aufgeführt werden. Rechtschreibe- und Zeichensetzungsfehler wurden korrigiert. Das Geschlecht oder andere persönliche Angaben des Coaches werden nicht offenbart.

\subsection{Stichprobenbeschreibung}

Die Coaches waren durchschnittlich 51 Jahre ( $S D=5,9$ Jahre) alt, hatten 6 Jahre ( $S D=2,1$ Jahre) Berufserfahrung als Coach. 50,3\% ihrer Arbeitszeit $(S D=24,4 \%)$ widmeten sie ihrer Arbeit als Coach. 37,5\% der Coaches waren männlich. Die Klienten waren im Schnitt 38 Jahre $(S D=7,0$ Jahre) alt und $12,5 \%$ der Klienten waren männlich. Durchschnittlich dauerten die Interviews $17 \min (S D=1,9 \mathrm{~min})$. 


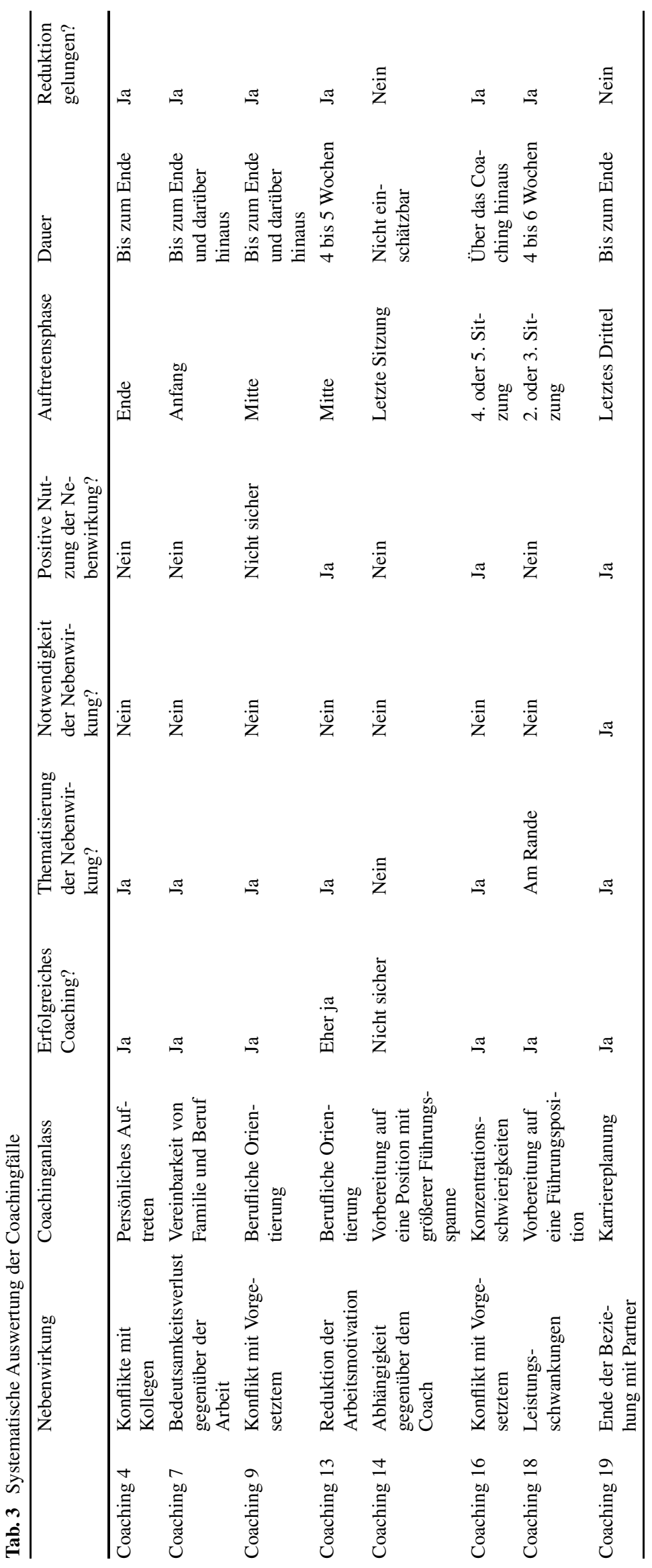




\section{Ergebnisse}

Zunächst sollen die einzelnen Fälle detailliert vorgestellt werden. Im Anschluss daran werden die Reaktionen der Coaches und Klienten auf die Nebenwirkungen beschrieben. Der Ergebnisteil schließt mit der Darstellung, wie die Nebenwirkungen bewältigt wurden.

\subsection{Vorstellung der Fälle}

Die fortlaufende Nummer aus den Transkripten wurde erhalten und wir beginnen die Beschreibung mit dem ersten Fall, der in die Auswertung einging.

\subsubsection{Fall 4: Konflikte mit Kollegen}

In diesem Fall gibt der Coach an, dass aufgrund des Coachings Konflikte zwischen den Kollegen und der Klientin aufgetreten seien. Die Klientin sei nach der Elternzeit zurück in ihr Unternehmen gekommen und habe mit ihm an ihrem persönlichen Auftreten und der Selbstsicherheit im Arbeitskontext gearbeitet: ,... wir haben dann stark an ihrem Zutrauen und ihrem Auftreten etc. gearbeitet und anfangs war sie da auch sehr froh und dankbar und angetan und so aber irgendwann ist es dann gekippt und sie hat dann eben auch, naja, eher mieses Feedback von den Kollegen bekommen. Also in dem Sinne dass sie eben zu selbstbewusst auftreten würde“ (Fall 4, S. 159-160).

\subsubsection{Fall 7: Bedeutsamkeitsverlust gegenüber der Arbeit}

Das Hauptthema von Fall 7 ist laut Coach die Vereinbarkeit von Familie und Beruf. Die Klientin habe mit ihm zufriedenstellender die privaten und beruflichen Herausforderungen lösen wollen. Der Coach beschreibt die Klientin als sehr gewissenhaft und schildert, dass sie den Sinn in ihrer Arbeit verloren habe: „Das klingt jetzt aber schon wirklich sehr dramatisch... aber doch so war es. Ihre Arbeitsmoral ist da deutlich den Bach runter gegangen, sie ist auch weniger gerne zur Arbeit gegangen und so“ (Fall 7, S. 180).

\subsubsection{Fall 9: Konflikt mit Vorgesetztem aufgrund starker Abgrenzung}

Anlass des Coachings ist eine berufliche Neuorientierung der Klientin, die sich in ihrer alten Position gestresst und unausgeglichen gefühlt habe. Durch Coaching 9 verschlechtert sich laut Aussage des Coaches die Beziehung zum Vorgesetzten: „Ich habe mal mit einem Coachee gearbeitet, bei der sich im Laufe des Coachings mehr oder minder leider gezeigt hat, dass sich die Beziehung zu ihrem Chef verschlechtert hat. Und ich denke, dass das an dem Punkt sehr deutlich auf das Coaching zurückzuführen war" (Fall
9, S. 191). Die Klientin habe gelernt sich abzugrenzen und eigene Prioritäten zu setzen, was für die Führungskraft neu gewesen sei und Konflikte produziert habe.

\subsubsection{Fall 13: Reduktion der Arbeitsmotivation}

Der Coach, der mit dem Klienten an einer beruflichen Neuorientierung gearbeitet hat, berichtet, dass es zu einem deutlichen Motivationsverlust bei seinem Klienten gekommen sei: „So kann man das schon sagen, also da war dann bei ihm ähm alle Motivation ähm irgendwie hin“ (Fall 13, S. 213). Laut Coach wollte der Klient wieder "on track“ gebracht werden, doch dann habe er ,noch mehr die Spur verloren“" (Fall 13, S. 213), was dann schwer gewesen sei.

\subsubsection{Fall 14: Abhängigkeit gegenüber dem Coach}

In diesem Coaching wird eine Klientin in eine Position mit größerer Führungsspanne gecoacht. Währenddessen kam es den Schilderungen nach zu einem Abhängigkeitsverhältnis zwischen Coach und Klientin: „Sie hat es beschrieben als würde ihr die Krücke genommen worden sein" (Fall 14, S. 220). Der Coach berichtet, dass der Effekt erst in der letzten Sitzung dem Coach und der Klientin richtig bewusst geworden sei. Die Klientin habe große Unsicherheit gespürt und sogar mehr Unsicherheit als vor dem Coaching.

\subsubsection{Fall 16: Konflikt mit Vorgesetztem}

Hier arbeiten Coach und Klient an den Konzentrationsschwierigkeiten des Klienten. Der Coach berichtet ebenfalls von einem Konflikt mit einem Vorgesetzten: „Mhm, in erster Linie war es wirklich einfach nur eine Verschlechterung der ähm ja Beziehungsebene. Also das habe ich aber tatsächlich auch schon öfters erlebt also mit den Vorgesetzten und auch wirklich oft mit den Kollegen. Also aus verschiedensten Gründen. Aber in diesem Fall war es also ja doch wirklich vor allem die Chefin“" (Fall 16, S. 231).

\subsubsection{Fall 18: Leistungsschwankungen}

In diesem Fall werden Leistungsschwankungen als eine Nebenwirkung des Coachings berichtet. Anlass des Coachings sei die erstmalige Übernahme einer Führungsaufgabe gewesen. Vor dem Coaching sei die Klientin sehr leistungsfähig gewesen und das in konstanter Ausprägung. Während des Coachings sei „,ihre Leistungsfähigkeit sehr, sehr schwankend gewesen" (Fall 18, S. 245).

\subsubsection{Fall 19: Ende der Beziehung mit Partner}

Der Coach in Fall 19 arbeitet mit der Klientin an der Planung ihrer Karriere. Es entsteht während des Coachings 
eine Nebenwirkung im privaten Bereich, die für die Klientin hochemotional gewesen sei. Die Klientin habe erkannt, sagt der Coach, dass ihr Partner sie in ihrer Entwicklung behindere. Die Klientin habe die Beziehung beendet, ,und ich bin mir da recht sicher, dass das in der Form zumindest nicht zu dem Zeitpunkt passiert wäre, wenn das Coaching da nicht gewesen wäre“" (Fall 19, S. 250).

Wie in Tab. 3 ersichtlich, werden alle Coachings bis auf das 14. Coaching als erfolgreich eingeschätzt. Beim 14. Coaching ist der Coach sich nicht sicher, ob es sich um ein erfolgreiches Coaching gehandelt habe. Bis auf das letzte Coaching sehen alle Coaches keine Notwendigkeit der Nebenwirkung. Sie glauben nicht, dass die Nebenwirkung eine erforderliche Begleiterscheinung des Prozesses war, um das Coaching erfolgreich abschließen zu können. In vier Fällen konnte die Nebenwirkung genutzt werden, um andere positive Wirkungen zu erzielen. In den anderen Fällen wird das nicht so gesehen oder es herrscht, wie bei Fall 9, Unsicherheit vor. Das zeitliche Auftreten fällt genauso heterogen wie die Dauer aus. Z. B. wird die Abhängigkeit (Fall 14) erst in der letzten Sitzung deutlich, während der Bedeutsamkeitsverlust (Fall 7) schon am Anfang auftritt. In drei Fällen bleibt die Nebenwirkung laut Einschätzung der Coaches auch über die letzte Sitzung hinaus existent (7, 9, 16). Bis auf ein Coaching (14) wurden alle Nebenwirkungen direkt thematisiert. Die Abhängigkeit konnte, laut Aussage des Coaches, nur schwer explizit thematisiert werden, weil sie so spät bemerkt wurde und es nicht möglich war, dass Coaching zu verlängern. In sechs von acht Fällen konnte der Effekt reduziert werden. Welche Verhaltensweisen und Rahmenbedingungen dazu beigetragen haben und wie Coaches und Klienten auf die Nebenwirkungen reagierten, soll in den nächsten Abschnitten berichtet werden.

\subsection{Wie reagieren Coaches und Klienten auf das Auftreten von Nebenwirkungen?}

Im Folgenden sollen die affektiven und kognitiven Reaktionen auf die Effekte beschrieben werden, die wir als $\mathrm{Ne}$ benwirkungen identifiziert haben.

\subsubsection{Fall 4: Konflikte mit Kollegen}

Bei diesem Fall wird die Nebenwirkung im Abschlussgespräch thematisiert. Als die Nebenwirkung zur Sprache kommt, sei die Klientin ärgerlich auf den Coach gewesen: „Sie war dann einfach, ja doch schon, also richtig böse auf mich“ (Fall 4, S. 160). Gleichzeitig wirkt sie auf den Coach verwirrt und überfordert von der Situation. Die Klientin habe dem Coach vorgeworfen, alles nur schlimmer gemacht und ihr falsche Methoden beigebracht zu haben. „Das war das wohl unangenehmste Abschlussgespräch, das ich je hatte", fasst der Coach das Gespräch zusammen (Interview 4,
S. 159). Der Coach gibt an, er habe mit Erstaunen reagiert und versucht, die Klientin zu verstehen, was ihm nicht vollständig gelungen sei. Gleichzeitig betont der Coach, dass er seine Gefühle strikt aus dem Coaching herausnehme.

\subsubsection{Fall 7: Bedeutsamkeitsverlust der Arbeit}

Hier reagiert laut Coach die Klientin auf den Bedeutsamkeitsverlust ihrer Arbeit mit Desillusionierung. Darauf folgen negative Gefühle wie Traurigkeit, Niedergeschlagenheit und Unsicherheit. „Naja sie hatte eben das Gefühl, dass das was sie tut, gar nicht so wichtig war, wie sie vorher immer dachte“ (Fall 7, S. 181). Der Coach benennt auf die entsprechende Frage keine Gefühle oder Gedanken. Er betont, dass er gelernt habe, seine Gefühle aus den Themen herauszunehmen und dass dies seinem Coachingverständnis entspreche.

\subsubsection{Fall 9: Konflikt mit Vorgesetztem aufgrund starker Abgrenzung}

Die Klientin aus dem neunten Fall reagiert auf die Konflikte mit dem Vorgesetzten mit gemischten Gefühlen. Laut Aussage des Coaches sei sie stolz auf sich selbst gewesen, weil sie es geschafft habe, sich von ihrer Arbeit abzugrenzen und „nein“ zu sagen. Gleichzeitig sei ihr wegen des Ärgers mit dem Vorgesetzten unwohl gewesen. Der Coach fühlt sich ähnlich hin und her gerissen. Er weiß nicht, wie sich die Situation entwickeln wird. Aus seiner Erfahrung weiß er, dass die neu entstehenden Konflikte manchmal herausfordernder als das eigentliche Problem sein können. Im Großen und Ganzen sei er aber froh gewesen, dass die Klientin sich so stark habe positionieren können.

\subsubsection{Fall 13: Reduktion der Arbeitsmotivation}

Der Klient aus dem diesem Fall, der während des Coachings noch stärker ,die Spur verliert“, reagiert laut Coach mit starken negativen Gefühlen. Der Klient habe sich vor allem niedergeschlagen gefühlt (,,Oh ja er war am Boden!“ Fall 13, S. 215). Er habe geweint, sich depressiv geäußert und alles in Frage gestellt. Der Coach benennt keine Gefühle und Gedanken, die er in dieser Situation gehabt habe. Er nehme sich zurück, weil seine Gefühle dort nichts zu suchen hätten und habe eher nach Lösungen in seinem Methodenkoffer gesucht.

\subsubsection{Fall 14: Abhängigkeit gegenüber dem Coach}

Als die Abhängigkeit in der letzten Sitzung dieses Coachings bemerkt wird und die Klientin glaubt, dass ihr die Krücke genommen werde, seien die Klientin und der Coach beide erstaunt gewesen. Für beide sei diese Erkenntnis über- 
raschend aufgetreten, insbesondere weil der Prozess so erfolgreich verlaufen sei. Dazu kommen auf der Seite der Klientin Traurigkeit und Überforderung. Der Coach offenbart seine Gefühle gegenüber der Klientin nicht: „Aber privat also so ganz für mich war ich definitiv durch den Wind. Habe mich gefragt inwiefern ich das hätte erkennen müssen“ (Fall 14, S. 221).

\subsubsection{Fall 16: Konflikt mit Vorgesetztem}

Der Coach beschreibt bezogen auf dieses Coaching, dass die Klientin „nicht mal sehr erstaunt oder erschrocken oder so etwas [war] sondern hat es ... ja sehr neutral aufgenommen“ (Interview 16, S. 233). Er führt das darauf zurück, dass er seine Klienten schon früh darüber aufkläre, dass Coaching kein Allheilmittel sei und Nebenwirkungen auftreten könnten. Der Coach macht aber klar, dass die neue Situation von der Klientin sehr wohl als Kraft raubend erlebt worden sei. Das Coachingverständnis des Coaches verbiete ihm Gefühle in einer solchen Situation zu haben. Er führt gegenüber der Interviewerin aber seine Gedanken in der Situation aus: „Veränderungen sind ja eigentlich nie frei von irgendwelchen sagen wir mal ungemütlichen Dingen und also meine Lebenserfahrung hat mir eigentlich also beigebracht, dass wenn sich etwas verändert die Reaktionen sich auch verändern. Also so gesehen ist das ja ganz natürlich. Ich bin da dann, ähm, also ganz und gar nicht erstaunt, wenn so eine Sache passiert. Also da ist Ihre Nebenwirkung wie Sie es nennen schon ein sehr passendes Wort. Alles hat eben Nebenwirkungen“" (Fall 16, S. 233).

\subsubsection{Fall 18: Leistungsschwankungen}

Im 18. Coaching kann der Coach nur wenige Informationen über die Reaktionen der Klientin mitteilen, da die Nebenwirkung nur am Rande thematisiert wurde. Sie glaubt dennoch, dass die Klientin verunsichert gewesen sei und ,auch daran gezweifelt hat, was das so bringen soll" (Interview 18, S. 246). Der Coach betont, dass er als Coach keine Gefühle habe. Die Gedanken seien aber zielorientiert gewesen. Sie hätten sich auf den Einsatz von Methoden bezogen, die dem Klienten in seiner Situation hätten helfen können.

\subsubsection{Fall 19: Ende der Beziehung mit Partner}

Laut Aussage des Coaches aus diesem Interview reagiert die Klientin auf das Ende der Beziehung mit großer Traurigkeit. Die Klientin habe viel geweint, aber nie den Zweifel gehabt, dass das Beziehungsende an dieser Stelle eine falsche Entscheidung gewesen sei. Der Coach gibt an, in der Situation mit starkem Mitgefühl reagiert zu haben. Aus Gründen der Professionalität habe sie aber diese Gefühle nicht gezeigt.

\subsection{Wie werden Nebenwirkungen bewältigt?}

Im Folgenden sollen die Verhaltensweisen von Coach und Klient, also die behaviorale Reaktion auf die Nebenwirkung, sowie die Rahmenbedingungen vorgestellt werden, die zur Bewältigung der Nebenwirkungen beigetragen haben. Da im 14. und 19. Fall die Coaches angaben, dass die Folgen der Nebenwirkungen nicht reduziert werden konnten, haben die Coaches hierzu keine Aussagen gemacht.

\subsubsection{Fall 4: Konflikte mit Kollegen}

Im diesem Fall versucht der Coach entkatastrophisierend auf die Klientin einzuwirken: „Na, ich hab halt die ganze Zeit versucht zu erläutern, dass das ja gar nichts Schlimmes ist" (Fall 4, S. 163). Er erläutert weiter, dass er der Klientin vermittelt habe, dass das so komme, wenn man sein Verhalten ändere und sich anders benimmt als alle anderen von einem bisher gewohnt seien. Diese Intervention habe er aber mehrmals wiederholen müssen, da die Klientin im Arbeitskontext immer wieder einen „Dämpfer“ erlebt habe. Die Klientin wandte eine eigene Strategie an, um die Nebenwirkung zu reduzieren. Laut Coach habe sie teilweise einfach wieder ihr vorheriges Verhalten gezeigt, um weniger negatives Feedback zu erhalten.

\subsubsection{Fall 7: Bedeutsamkeitsverlust gegenüber der Arbeit}

Hier gibt der Coach bei der Frage nach der Bewältigung des Effektes an, in seiner Rolle geblieben zu sein. Er habe Verständnis gezeigt, wäre offen mit der Situation umgegangen und hätte der Klientin Raum gelassen. Auf der Seite der Klientin sieht er als hilfreich an, dass diese auch die Vorteile der neuen Situation erkannt habe und bei den Rahmenbedingungen, dass ihre Familie sich durchaus gefreut habe, dass die Klientin nun mehr Zeit habe.

\subsubsection{Fall 9: Konflikt mit Vorgesetztem aufgrund starker Abgrenzung}

Beim neunten Fall versucht der Coach dem Klienten einen differenzierten Blick auf die Konfliktsituation zu ermöglichen. Die Klientin solle keine Angst vor dem Konflikt haben, diesen aber auch nicht auf die leichte Schulter nehmen oder ihn herausfordern. Auf Seiten der Klientin sei hilfreich gewesen, dass sie diese Punkte gut angenommen habe und die Situation beruhigt werden konnte. Zusätzlich habe sie das Gespräch mit ihrem Vorgesetzten gesucht, was durchaus hilfreich gewesen sei. Bei den Rahmenbedingungen wurden zwei Aspekte durch den Coach angesprochen. Zum einen habe die Klientin großen Rückhalt bei den Kollegen erhalten. Dieser Rückhalt habe ihr geholfen ,ihrer neuen Linie treu zu bleiben und dann eben auch die Kraft zu haben, of- 
fensiv damit umzugehen und sich ihrem Chef zu erklären“" (Fall 9, S. 194-195). Als weitere positive Rahmenbedingung wurde der Vorgesetzte genannt, der sich kooperativ verhalten habe.

\subsubsection{Fall 13: Reduktion der Arbeitsmotivation}

In diesem Fall versucht der Coach dem Thema aktiv die „Wichtigkeit“ bzw. die „Schwere“ zu nehmen (13. Fall, S. 217). Besonders gut geholfen hätten Auflistungen, was wichtig im Leben sei und was nicht. Auf der Seite des Klienten wird als hilfreich erachtet, dass der Klient mitgemacht und nicht gemauert habe. Bei den Rahmenbedingungen wird der Kontakt zu freundlichen Kollegen genannt, was motivationsförderlich gewirkt habe.

\subsubsection{Fall 16: Konflikt mit Vorgesetztem}

Hier gibt der Coach an, Techniken des Perspektivwechsels genutzt zu haben. Er versucht damit das Verständnis und damit die Akzeptanz der Klientin für die Situation zu erhöhen. Auf Klientenseite bemerkt er eine hohe Offenheit. Die Klientin sei ergebnisoffen sowie selbstverantwortlich gewesen und habe niemanden verurteilt. Bei den Rahmenbedingungen erwähnt der Coach die Chefin. Er lobt deren fachliche aber auch soziale Kompetenz (insbesondere die Kommunikations- und Konfliktfähigkeit).

\subsubsection{Fall 18: Leistungsschwankungen}

Auch beim 18. Fall arbeitet der Coach an der kognitiven Neubewertung der Situation. Er nimmt der Klientin die Unsicherheit, indem er ihr vermittelt, ,dass das dazugehören kann, aber dass das jetzt auch nicht so bleibt" (18. Fall, S. 248). Weiterhin setzt er Übungen zur Förderung der Konzentrationsfähigkeit ein. Bei der Klientin hebt der Coach hervor, dass es wichtig gewesen sei, dass sie ihm ein großes Vertrauen entgegengebracht habe. Auch sei der Prozess sehr offen und die Atmosphäre zwischen Coach und Klientin positiv gewesen.

\section{Diskussion}

Die Einleitung und der Forschungsüberblick zeigten, dass Coachings positive mittelgroße Effekte bewirken. Gleichzeitig werden Nebenwirkungen regelmäßig von Coaches und Klienten wahrgenommen. Es treten vielfältige Nebenwirkungen auf, die das psychische Wohlbefinden, die soziale Integration, die Leistungsfähigkeit, die Bewertung der Arbeitsrolle oder materielle Verluste des Klienten betreffen können. Die Nebenwirkungen besitzen eine eher niedrige bis mittlere Intensität und schwerwiegendere Nebenwirkun- gen treten selten auf. Da die bisherigen Ergebnisse mehrheitlich quantitativ gewonnen wurden, wurden in der vorliegenden Studie einzelne Coachingfälle qualitativ vorgestellt. Diese sollen im Folgenden diskutiert werden.

Gegenüber der von Schermuly et al. (2014) explorierten Liste traten keine neuen Nebenwirkungen auf. Nutzt man das oben vorgestellte Kategoriensystem, dann sind fünf der Nebenwirkungen der Kategorie soziale Integration zuzuordnen $(4,9,14,16,19)$. Durch Coachings werden Veränderungen angestoßen. Wie die Fallbeispiele zeigen, entwickeln sich durch die Coachings neue Perspektiven und der Klient wird zu neuen Verhaltensweisen angeregt. Die Führungskräfte und Kollegen werden aber nicht gecoacht, vermutlich wissen viele noch nicht einmal davon und so verändert sich das soziale Umfeld des Klienten nicht synchron. Das Umfeld verharrt in den alten Erwartungen an den Klienten und seine Rolle in der Organisation. Es kommt zu Irritationen auf beiden Seiten, die in Konflikten münden können. Rollen und Beziehungen müssen neu definiert werden, was für die Beteiligten eine Herausforderung darstellt.

In die Kategorie Leistungsfähigkeit gehören die Nebenwirkungen aus Coaching 13 und Coaching 18. Durch ein Coaching kann es zu Leistungsschwankungen kommen. Der interviewte Coach hat dafür auch eine Erklärung: „... vielleicht hat sie sich eben $\mathrm{zu}$ sehr auf die Themen aus dem Coaching konzentriert und dann darüber eben den Fokus bei anderen Dingen verloren“ (Fall 18, S. 247). Ein Coaching kann die Aufmerksamkeit eines Klienten okkupieren. Neues (Rollen-)verhalten einzuüben beansprucht Zeit und kostet Energie. Darunter können unter Umständen andere Arbeitsprozesse leiden. Auch ist es möglich, dass Veränderungen, die ein Coaching bewirkt, mit Anpassungsproblemen im Arbeitskontext einhergehen, die vorübergehend die Leistungen senken oder schwanken lassen (Schermuly 2016 ).

Die Nebenwirkung aus Fall 7 gehört in die Kategorie Bewertung der Arbeitsrolle. Durch das Coaching erlebt die Klientin ihre Arbeit als weniger bedeutsam und leidet auch darunter. Fall 7 verdeutlicht auch gut, dass Nebenwirkungen für unterschiedliche Personen unterschiedliche Valenz besitzen. Während der Effekt von der Klientin als unerwünscht und negativ erlebt wird, freuen sich die Angehörigen über eine Konsequenz der Nebenwirkung. Die Klientin hat wieder mehr Zeit für ihre Familie, weil sie sich weniger für ihren Beruf engagiert.

Wie bereits quantitativ abgesichert (Schermuly et al., 2014), zeigen die Interviews, dass Nebenwirkungen nicht mit Misserfolg gleichzusetzen sind. Kein Coaching wird von den Coaches eindeutig als nicht erfolgreich bezeichnet. Lediglich ein Coach ist sich nicht sicher, ob das Coaching erfolgreich war. Gleichzeitig wird die Nebenwirkung von sieben der acht Coachings als nicht notwendig für die Erreichung des Coachingerfolgs bezeichnet. Erneut bestätigt 
sich, dass Nebenwirkungen auch Bestandteil von erfolgreichen Coachings sein können. Sie helfen aber überwiegend nicht bei der Zielerreichung. So sagt Coach 4 „Also das wäre ja sogar noch viel einfacher gewesen das alles gut abzuschließen, wenn sie da jetzt keine Verwirrung gehabt hätte" (Fall 4, S. 163) oder Coach 9, ,... ich denke, dass sie das auch ohne den Streit mit ihrem Chef erreicht hätte" (Fall 9, S. 194). Doch können die Effekte zumindest in drei Fällen positiv genutzt werden. Durch die Nebenwirkung konnte das Thema gründlich und tief in Fall 13 bearbeitet werden. Ähnlich wird der Konflikt mit dem Vorgesetzten in Fall 16 als Möglichkeit gesehen, sich stärker mit dem Thema auseinanderzusetzen und die eigene Position zu stärken. Durch die Trennung sei das Leben der Klientin auf den Kopf gestellt worden und sie hätte endlich machen können, was sie wolle (Fall 19). Diese Ergebnisse ermöglichen eine neue Perspektive auf Nebenwirkungen. Nebenwirkungen scheinen nicht notwendig zu sein, um Coachings erfolgreich abschließen zu können. Sie liefern aber manchmal eine Chance für z.B. eine tiefere Verarbeitung von Themen und die Stärkung einer Position. Zukünftige Forschung sollte hier ansetzen und analysieren, wann und warum manchmal Nebenwirkungen nützlich sein können und dennoch keinen Einfluss auf den Coachingerfolg besitzen.

Die Klienten reagieren mit unterschiedlichen Emotionen auf die Nebenwirkungen. Dies ist nicht überraschend, da es sich um unterschiedliche Nebenwirkungen und verschiedene Menschen handelt. Dazu gehören Ärger (Fall 14), Traurigkeit/Niedergeschlagenheit $(7,13,14,19)$, Verunsicherung (18), Überforderung (14), Unwohlsein (9) und Stolz (9). Ordnet man die Emotionen den Sektoren des Circumplexes zum affektiven Wohlbefinden bezogen auf den Arbeitskontext von Warr (1994, S. 85) zu, der durch die Achsen Wohlbefinden und Erregung aufgespannt wird, dann sind bis auf eine Emotion (Stolz) alle dem negativen Wohlbefinden zuzuordnen. Die meisten Emotionen (ausgenommen Ärger und Stolz) gehören in den Sektor „,depressed“, der durch negatives Wohlbefinden und niedrige Erregung gekennzeichnet ist. Nebenwirkungen wirken sich demnach vor allem negativ auf das psychische Wohlbefinden der Klienten aus.

Die Coaches reagieren mit Erstaunen $(4,14)$, sind ganz schön durch den Wind und haben Selbstzweifel (14). Sie sind hin- und hergerissen (9) und teilweise froh über die Nebenwirkung (9). Viele Coaches betonen aber, aufgrund ihrer Coachrolle keine Gefühle zu haben oder haben zu dürfen bzw. ihre Gefühle herauszunehmen $(4,7,13,16,18)$. Sehr deutlich bringen das Coach 16 und 18 zum Ausdruck: „Also, naja mein Verständnis von Coaching verbietet mir das. Die Gefühle des Coaches haben da nichts zu suchen“" (Fall 16, S. 233); ,also als Coach habe ich da keine Gefühle, das ist ja ähm also vollkommen gegen das was ich als Coaching-Position so gelernt habe. Ähm aber... also als
Mensch klar da fragt man sich schon was da dann jetzt so der Auslöser war und ob es an einem oder also an der eigenen Arbeit hängt“" (Fall 18, S. 246). Die Ablehnung von Gefühlen überrascht, da Coaches wie die Forschung zu Nebenwirkungen von Coaching für Coaches zeigt, sehr häufig auch mit negativen Gefühlen während der Coachingprozesse konfrontiert sind. So fühlen sich Coaches in über $40 \%$ der Coachings zumindest etwas persönlich betroffen durch ein Thema oder hatten Angst der Coachrolle nicht gerecht zu werden (Schermuly 2014).

Cox und Bachkirova (2007) analysierten in ihrer qualitativen Studie den Umgang mit schwierigen emotionalen Situationen in Coachings bei 39 Coaches aus Großbritannien. Die Autoren identifizierten drei verschiedene Bewältigungsstrategien, wenn Coaches mit eigenen unangenehmen Emotionen in einem Coachingprozess konfrontiert werden. Während einige Coaches über ihre Emotionen reflektieren oder diese in die Supervision tragen, thematisieren andere diese direkt mit den Klienten und nutzen sie aktiv für den Coachingprozess. Als dritte Strategie wird die Beendigung des Coachings genannt, insbesondere wenn die negativen Emotionen zu stark sind (Cox und Bachkirova 2007). Die Strategien der in unserer Studie zitierten Coaches könnten die Nutzung der beschriebenen Strategien erschweren. Wenn Coaches ihren Gefühlen im Coachingprozess keine Beachtung schenken oder sie ablehnen (,als Coach habe ich da keine Gefühle“, siehe Fall 18 weiter oben), können sie weder über diese reflektieren (z. B. im Rahmen der Supervision) noch diese aktiv im Coachingprozess nutzen. Einfühlungsvermögen und emotionale Kompetenz gehören zu den wichtigsten Coachkompetenzen (Lai und McDowall 2014; Schiessler 2010; Theeboom et al. 2014). Dazu gehört nicht nur die Wahrnehmung, das Verstehen und die Regulation der Gefühle der Gegenüber sondern auch der eigenen Gefühle (Mayer et al. 2004). Bezogen auf die Gefühle der Klienten und die des Coaches schlussfolgern Lai und McDowall (2014, S. 127): „Thus, managing these emotions and transferring them into positive insights for coachees to change is a crucial factor for an effective coaching relationship“. Die Besonderheiten im Umgang mit Gefühlen dieser Coaches, wirft die Frage auf, welche Ausbildung die Coaches absolviert haben. Z. B. begründet der Coach in Fall 18 seine Gefühlsabstinenz mit dem, was er gelernt habe. Leider können wir diese Frage nicht beantworten, denn in unseren Interviews wurde der Ausbildungshintergrund nicht abgefragt, was wir auch in den Limitationen als Fehler verdeutlichen.

Eine Art Mittelweg geht hier Coach 19: „Ich hatte da schon wirklich starkes Mitgefühl. Also so eine Situation ist natürlich wirklich hochemotional. Da flossen bei ihr auch viele, viele Tränen wirklich. Ähm ja, ich meine auch wenn ich da dann mitfühle, das zeige ich natürlich nicht. Also da bin ich schon sehr professionell“. Der Coach lässt sein 
Mitgefühl zu, doch zeigt er es aus Gründen der Professionalität der Klientin nicht. Mehr Forschung ist notwendig, wie Emotionen von Coach und Klient in Wechselwirkung zueinander stehen und wie die gezeigten Gefühle des Coaches den Coachingprozess beeinflussen.

Z. B. fordert die klientenzentrierte Beratung Echtheit und Kongruenz von ihren Therapeuten (Rogers, 1993). Aus der Therapieforschung ist weiter bekannt, dass die emotionale Öffnung (self-disclosure) des Therapeuten positive Effekte auf den Klienten hat, wenn die Beziehungsqualität hoch ist, aber negativ wirkt, wenn diese niedrig ist (Henretty und Levitt 2010). Mit der Öffnung kann der Therapeut als Rollenbild für den Klienten fungieren und diesen zu ähnlichem Verhalten bewegen (Henretty et al., 2014).

Die meisten Nebenwirkungen konnten in den Coachings bewältigt werden. Damit dies gelingt, scheint es wichtig zu sein, dass der Effekt früh genug durch den Coach wahrgenommen wird. In Coaching 14 wird die Abhängigkeit erst in der Abschlusssitzung entdeckt. Dies ist zu spät, um den Effekt noch reduzieren oder positiv nutzen zu können.

Mehrere Coaches arbeiten mit den Klienten an einer kognitiven Neubewertung der Situation, um den Bedrohungscharakter zu reduzieren $(4,13,16,18)$. Sie machen den Klienten deutlich, dass die Nebenwirkung normal sei und dazu gehöre. Coach 18 vermittelt der Klientin zusätzlich, dass die Nebenwirkung nur einen temporären Charakter besitzt. Dies ähnelt sehr dem weiter oben berichteten Forschungsstand: Nebenwirkungen sind normal und haben zumeist eine zeitlich beschränkte Wirkung. Einer normalen und zeitlich begrenzten unerwünschten Wirkung ausgesetzt zu sein, vermittelt mehr Kontrolle über die Situation und reduziert deren Bedrohlichkeit. Kontrolle hat sich in vielen Studien als entscheidender Faktor zu Reduzierung von Stress bewährt (siehe z. B. exemplarisch das Demand Control Modell, Karasek 1979 oder das psychologische Empowermentmodell, Spreitzer 1995). Coach 16 hat die Klientin sogar proaktiv auf die Nebenwirkungen vorbereitet, so dass die Klientin von der Nebenwirkung nicht überrascht ist. Ansonsten zeigen die Coaches Verständnis (7), vermitteln ein differenziertes Bild der Konfliktsituation (9) und nutzen Techniken des Perspektivwechsels (16). All diese Interventionen werden durch die direkte Thematisierung und einen grundsätzlich offenen Umgang mit der Nebenwirkung erleichtert.

Auf Seiten der Klienten werden die Offenheit $(13,16)$, die Selbstverantwortlichkeit und (Pro)Aktivität des Klienten $(9,13,16)$ als wichtige Aspekte der Bewältigung wahrgenommen. So sucht z. B. die Klientin im 9. Fall das direkte Gespräch mit ihrem Vorgesetzten und hat damit Erfolg. Weiterhin wird von einem Coach das entgegengebrachte Vertrauen (18) als wichtiger Faktor der Bewältigung wahrgenommen. Die Beziehungsqualität scheint nicht nur wichtig für das Verhindern von Nebenwirkungen zu sein sondern auch für deren erfolgreiche Bewältigung. Weiterhin hatte die Wahrnehmung der positiven Konsequenz der Nebenwirkungen (mehr Zeit für die Familie) in einem Coaching einen relevanten Einfluss (7). Es tritt aber zumindest eine Bewältigungsstrategie auf, die vom Coach als dysfunktional erlebt wird. Um die Nebenwirkung zu reduzieren, zeigte die Klientin wieder ihr altes Verhalten.

Bei den Rahmenbedingungen tritt insbesondere das soziale System als Ressource auf. Die Klientin aus dem 9. Fall wird während des Konfliktes von den Kollegen unterstützt (freundliche Kollegen helfen auch im 13. Fall). Dazu kommt eine kooperative Führungskraft. Die Bedeutung der Führungskraft wird auch bei Fall 16 betont. Die Führungskraft besitzt eine hohe soziale Kompetenz, die es ihr ermöglicht, positiv auf die Situation einzuwirken. Zukünftige Forschung sollte die komplexen sozialen Wirkungen von Nebenwirkungen am Arbeitsplatz weiter aufklären und dazu mehrere Interaktionspartner im sozialen System befragen.

\subsection{Praktische Implikationen}

Kilburg schlussfolgerte 2002 (S. 288) mit Blick auf das Thema unerwünschte Effekte im Coaching: „Despite the importance of knowing how to manage these issues, there is virtually nothing available in the literature to help executive coaches face these problems." Die aktuelle Forschung hat geholfen, diese Lage zu verbessern. Es ist bekannt, welche Nebenwirkungen wie oft von Klienten und Coaches wahrgenommen werden und welche Ursachen für diese beobachtet werden. Durch die qualitativen Ergebnisse ist zusätzlich für einige Nebenwirkungen bekannt geworden, wie diese konkret wirken und wie sie bewältigt werden können. Diese Ergebnisse sollten in der Ausbildung von Coaches genutzt werden, was bisher noch nicht geschieht. Möller und Hellebrandt (2015) untersuchten die wissenschaftliche Fundierung von Coachingausbildungen in Deutschland. Den Ergebnissen nach berücksichtigte keine der in Deutschland untersuchten 33 Ausbildungen das Thema Nebenwirkungen von Coaching. Hier herrscht Nachholbedarf. Durch die Integration des Nebenwirkungsthemas kann die Coachingausbildung professionalisiert werden. Gleichzeitig können sich damit Ausbildungsinstitute von ihren derzeit 250 Mitbewerbern in Deutschland abheben.

Für die Bewältigung von Nebenwirkungen scheint es hilfreich zu sein, wenn die Coaches diese frühzeitig bemerken. Deswegen sollten Coaches den Coachingprozess aufmerksam mit Blick auf Nebenwirkungen verfolgen. Weiterhin deuten die Ergebnisse darauf hin, dass eine hohe Beziehungsqualität nicht nur positive Effekte bewirkt sondern auch Nebenwirkungen einschränkt und, wenn sie auftreten, bei der Bewältigung hilft. So geht Bluckert (2005) davon aus, ,that the coaching relationship is not just a critical 
success factor, but arguably the critical success factor in successful coaching outcomes" (S. 336). So konnte bereits gezeigt werden, dass die Beziehungsqualität einen positiven Einfluss auf die Zufriedenheit mit dem Coaching, die wahrgenommene Nützlichkeit des Coachings, die Leistung des Klienten und auch auf die Gesamtevaluation des Coachings hat (Boyce et al. 2010). Coaches sollten sich also aktiv um eine gute Beziehungsqualität zu ihren Klienten bemühen. Ob dabei das in der vorliegenden Studie offenbarte Gefühlsverbot mancher Coaches hilfreich ist, bleibt fraglich.

Grundsätzlich scheint sich ein offener und entspannter Umgang mit Nebenwirkungen zu lohnen. Der Coach aus dem 16. Coaching bringt es auf den Punkt: Veränderungen sind nicht frei von ungemütlichen Begleiterscheinungen. Wenn Coaches diese als normale Begleiter des Coachings wahrnehmen, dann können das auch Klienten. Der Coach dient als Modell für den Umgang mit Nebenwirkungen. So findet eine Entkatastrophisierung statt. Dadurch ergibt sich die Chance, die Nebenwirkungen als Thema in das Coaching zu integrieren, eine kognitive Neubewertung der Situation zu erreichen und manchmal sogar positive Konsequenzen der Nebenwirkung zu nutzen und damit zusätzliche Ziele zu erreichen.

Obwohl die befragten Coaches nicht explizit die Supervision ansprachen, erscheint sie dennoch als eine sinnvolle Möglichkeit, um Nebenwirkungen bewältigen zu können. Eine zentrale Funktion der Supervision ist die professionelle Unterstützung bei der Problembewältigung und -klärung (Möller und Kotte 2015). Im Rahmen von Supervision könnten somit aufgetretene Nebenwirkungen direkt thematisiert und Möglichkeiten zu deren Bewältigung besprochen werden. So gaben Coaches in einer Befragung von Passmore und McGoldrick (2009) an, dass sie Supervision als Möglichkeit nutzen, um mit Herausforderungen, die sie im Coaching erlebt haben, besser umzugehen. In der Studie von Schermuly et al. (2014) gaben 10,7\% der Coaches an, dass fehlende Supervision sogar eine Ursache für aufgetretene Nebenwirkungen war. Supervision erscheint daher als geeignete Möglichkeit zur Vermeidung und Bewältigung von Nebenwirkungen. Nichtsdestotrotz ist weitere Forschung notwendig, um die Wirkung von Supervision bei Coaches konkreter zu untersuchen.

\subsection{Limitationen}

Qualitative Studien sind durch kleine Stichproben geprägt, so dass eine Generalisierung der Ergebnisse nicht zulässig ist. Die Repräsentativität ist zusätzlich dadurch eingeschränkt, dass bei der Stichprobenziehung ein Bias entstanden sein kann. Das Forschungsthema wurde transparent bei der Einladung der Teilnehmer vorgestellt. Der professionelle Umgang mit Nebenwirkungen, der in den In- terviews ersichtlich ist, kann dadurch beeinflusst worden sein, dass nur Coaches an einer Studie zu Nebenwirkungen von Coaching teilnehmen, die bereits einen professionellen Umgang mit dem Thema pflegen. Eine weitere methodische Schwäche besteht darin, dass nur Interviews mit den Coaches geführt wurden. Zukünftige Forschung sollte gleichzeitig auch die Klientenperspektive und die Perspektive von Interaktionspartnern (Führungskräften/ Kollegen) berücksichtigen. Weiterhin können demografische Aspekte der Coaches eine Einschränkung für die Repräsentativität darstellen. Der durchschnittliche Coach in Deutschland ist 50,9 Jahre alt und verbringt 32,4\% seiner Arbeitszeit mit Coaching (Middendorf, 2015). Die vorliegende Stichprobe ist deutlich jünger $(M=38$ Jahre) und besitzt dadurch nur 6 Jahre Berufserfahrung als Coach. Dafür liegt der prozentuale Anteil von Coaching an der Arbeitszeit $(50,3 \%)$ höher. Es handelt sich um eine eher unerfahrene Stichprobe, die aber aktuell viel coacht. Dieses Stichprobenartefakt kann die Ergebnisse beeinflusst haben. Darüber hinaus ist es als problematisch mit Blick auf den Umgang mit Gefühlen zu sehen, dass wir nicht erhoben haben, welche Coachingausbildung die Coaches absolviert haben. Unter Umständen kann die Ausrichtung einer Coachingausbildung diese spezielle Haltung erklären. Weiterhin sollten zukünftige qualitative Forschungen stärker die Ursachen der Nebenwirkungen betrachten. Die wahrgenommene Ursache einer Nebenwirkung kann unter Umständen den Umgang mit dieser stark beeinflussen. Z. B. macht es einen Unterschied für den Umgang, ob der Klient den Coach oder das Verfahren Coaching als ursächlich betrachtet.

Interessenkonflikt C. C. Schermuly und C. Graßmann geben an, dass kein Interessenkonflikt besteht.

Open Access Dieser Artikel wird unter der Creative Commons Namensnennung 4.0 International Lizenz (http://creativecommons.org/ licenses/by/4.0/deed.de) veröffentlicht, welche die Nutzung, Vervielfältigung, Bearbeitung, Verbreitung und Wiedergabe in jeglichem Medium und Format erlaubt, sofern Sie den/die ursprünglichen Autor(en) und die Quelle ordnungsgemäß nennen, einen Link zur Creative Commons Lizenz beifügen und angeben, ob Änderungen vorgenommen wurden.

\section{Literatur}

Baron, L., \& Morin, L. (2009). The coach - coachee relationship in executive coaching: a field study. Human Resource Development Quarterly, 20(1), 85-106.

Bennett, J.L. (2006). An agenda for coaching-related research: a challenge for researchers. Consulting Psychology Journal: Practice and Research, 58(4), 240-249.

Bluckert, P. (2005). Critical factors in executive coaching - the coaching relationship. Industrial and Commercial Training, 37(7), 336-340.

Boyce, L., Jackson, R., \& Neal, L. (2010). Building successful leadership coaching relationships: examining impact of matching cri- 
teria in a leadership coaching program. Journal of Management Development, 29(10), 914-931.

Brüsemeister, T. (2008). Qualitative Forschung: Ein Überblick. Wiesbaden: VS.

Cox, E., \& Bachkirova, T. (2007). Coaching with emotion: How coaches deal with difficult emotional situations. International Coaching Psychology Review, 2(2), 178-189.

Duck, S., \& Wood, J. T. (1995). For better, for worse, for richer, for poorer: the rough and the smooth of relationships. In S. Duck \& J. T. Wood (Hrsg.), Confronting relationship challenges (S. 1-21). Thousand Oaks: SAGE.

Eby, L. T., \& Allen, T. D. (2002). Further investigation of protégés' negative mentoring experiences: patterns and outcomes. Group and Organization Management, 27(4), 456-479.

Feldman, D. C., \& Lankau, M. J. (2005). Executive coaching: a review and agenda for future research. Journal of Management, 31(6), 829-848.

Flanagan, J.C. (1954). The critical incident technique. Psychological Bulletin, 51, 327-358.

Graßmann, C., \& Schermuly, C. C. (2016). Side effects of business coaching and their predictors from the coachees' perspective. Journal of Personnel Psychology. Advance online publication. doi:10.1027/1866-5888/a000161

De Haan, E., Duckworth, A., Birch, D., \& Jones, C. (2013). Executive coaching outcome research: the contribution of common factors such as relationship, personality match, and self-efficacy. Consulting Psychology Journal: Practice and Research, 65(1), 40-57.

Henretty, J.R., \& Levitt, H.M. (2010). The role of therapist selfdisclosure in psychotherapy: a qualitative review. Clinical Psychology Review, 30(2), 63-77.

Henretty, J. R., Currier, J. M., Berman, J. S., \& Levitt, H. M. (2014). The impact of counselor self-disclosure on clients: a meta-analytic review of experimental and quasi-experimental research. Journal of Counseling Psychology, 61(2), 191-207.

Jones, R.J., Woods, S. A., \& Guillaume, Y.R.F. (2015). The effectiveness of workplace coaching: a meta-analysis of learning and performance outcomes from coaching. Journal of Occupational and Organizational Psychology, 89(2), 249-277.

Karasek Jr, R. A. (1979). Job demands, job decision latitude, and mental strain: implications for job redesign. Administrative Science Quarterly, 24(2), 285-308.

Kilburg, R. R. (1996). Toward a conceptual understanding and definition of executive coaching. Consulting Psychology Journal: Practice and Research, 48(2), 134-144.

Kilburg, R. R. (2002). Failure and negative outcomes: the taboo topic in executive coaching. In C. Fitzgerald \& J. G. Berger (Hrsg.), Executive coaching: practices and perspectives (S. 283-301). Palo Alto: Davis-Black.

Kotte, S., Hinn, D., Oellerich, K., \& Möller, H. (2016). Der Stand der Coachingforschung: Kernergebnisse der vorliegenden Metaanalysen. Organisationsberatung, Supervision, Coaching, 23(1), 5-23.

Lay, Y.-L., \& McDowall, A. (2014). A systematic review of coaching psychology: focusing on the attributes of effective coaching psychologists. International Coaching Psychology Review, 9(2), 118-134.

Mayer, J.D., Salovey, P., \& Caruso, D. R. (2004). Emotional intelligence: theory, findings, and implications. Psychological Inquiry, 15(3), 197-215.
Middendorf, J. (2015). Wirtschaftlichkeit versus Vertraulichkeit? Daten zum Coaching-Markt Deutschland. Erdinger Coachingkongress, Erding. Vortrag. http://www.coaching-kongress.com/wpcontent/uploads/Pr\%C3\%A4sentation-Middendorf_komp. Zugegriffen: 5. Jan. 2016.

Mohr, D.C. (1995). Negative outcome in psychotherapy: a critical review. Clinical Psychology: Science and Practice, 2(1), 1-27.

Möller, H., \& Hellebrandt, M. (2015). Coaching-Weiterbildung: Jenseits der Wissenschaft. Wirtschaftspsychologie aktuell, 22(3), 9-12.

Möller, H., \& Kotte, S. (2011). Die Zukunft der Coachingforschung. Organisationsberatung, Supervision, Coaching, 4, 445-456.

Möller, H., \& Kotte, S. (2015). Supervision - past, present, future. Psychotherapie Im Dialog, 16(1), 16-24. Themenheft Supervision.

Oellerich, K. (2016). Negative Effekte von Coaching und ihre Ursachen aus der Perspektive der Organisation. Organisationsberatung, Supervision, Coaching, 23(1), 43-58.

Passmore, J., \& McGoldrick, S. (2009). Super-vision, extra-vision or blind faith? A grounded theory study of the efficacy of coaching supervision. International Coaching Psychology Review, 4(2), $143-159$.

Pogge, T. (2015). Negative Effekte von Coaching für den Klienten. Unveröffentlichte Masterarbeit, SRH Hochschule Berlin.

Ramos-Sánchez, L., Esnil, E., Goodwin, A., Riggs, S., Touster, L. O., Wright, L. K., Ratanasiripong, P., \& Rodolfa, E. (2002). Negative supervisory events: effects on supervision and supervisory alliance. Professional Psychology: Research and Practice, 33(2), 197-202.

Rogers, C. R. (1993). Die klientenzentrierte Gesprächspsychotherapie. Frankfurt: Fischer.

Schermuly, C.C. (2014). Negative effects of coaching for coaches: an explorative study. International Coaching Psychology Review, 9(2), 167-182.

Schermuly, C.C. (2016). Nebenwirkungen von Coaching für Klienten - Definition, Häufigkeiten, Kategorien und Ursachen. In C. Triebel, J. Heller, B. Hauser \& A. Koch (Hrsg.), Qualität im Coaching (S. 205-214). Berlin: Springer.

Schermuly, C.C., Schermuly-Haupt, M.-L., Schölmerich, F., \& Rauterberg, H. (2014). Zu Risiken und Nebenwirkungen lesen Sie ... - Negative Effekte von Coaching. Zeitschrift für Arbeits- und Organisationspsychologie, 58(1), 17-33.

Schiessler, B. (2010). Coaching als Maßnahme der Personalentwicklung. Berlin: Springer.

Seiger, C. \& Künzli, H. (2011). Der Schweizerische Coachingmarkt aus der Sicht von Coaches. Zürcher Hochschule für Angewandte Wissenschaften. http://pd.zhaw.ch/hop/544183222.pdf. Zugegriffen: 10. Juli 2013.

Spreitzer, G. M. (1995). Psychological empowerment in the workplace: dimensions, measurement, and validation. Academy of Management Journal, 38(5), 1442-1465.

Theeboom, T., Beersma, B., \& van Vianen, A.E. M. (2014). Does coaching work? A meta-analysis on the effects of coaching on individual level outcomes in an organizational context. The Journal of Positive Psychology, 9(1), 1-18.

Warr, P. (1994). A conceptual framework for the study of work and mental health. Work \& Stress, 8(2), 84-97. 